\title{
2723. Feature extraction method based on VMD and MFDFA for fault diagnosis of reciprocating compressor valve
}

\author{
Yan Liu ${ }^{1}$, Jindong Wang ${ }^{2}$, Ying $\mathrm{Li}^{3}$, Haiyang $\mathrm{Zhao}^{4}$, Shuxin $\mathrm{Chen}^{5}$ \\ 1, 2,3,4 Mechanical Science and Engineering Institute, Northeast Petroleum University, Daqing, China \\ ${ }^{1,5}$ Mechanical and Electrical Engineering Institute, Qiqihar University, Qiqihar, China \\ ${ }^{2}$ Corresponding author \\ E-mail: 1liuyan1977@126.com, ${ }^{2} w j d 327 @ 126 . c o m,{ }^{3}$ liyingnpu@126.com, ${ }^{4}$ zhaohaiyang2003@126.com, \\ 545102276@qq.com
}

Received 6 June 2017; received in revised form 7 September 2017; accepted 28 November 2017 DOI https://doi.org/10.21595/jve.2017.18726

Check for updates Abstract. Aiming at the nonlinearity, nonstationarity and multi-component coupling
characteristics of reciprocating compressor vibration signals, an integrated feature extraction
method based on the variational mode decomposition (VMD) and multi-fractal detrended
fluctuation analysis (MFDFA) is proposed for a fault diagnosis for a reciprocating compressor
valve. Firstly, to eliminate the noise interference, a novel VMD method with superior
anti-interference performance was utilized to obtain several components of the quasi-orthogonal
band-limited intrinsic mode function (BLIMF) from a strong non-stationarity vibration signal, and
a consistent number K of BLIMFs was selected based on a novel criterion for all fault states.
Secondly, the MFDFA method, which can describe the multi-fractal structure feature of
non-stationary time series, was applied to analyze each BLIMF component, and the parameters of
MFDFA were employed as the eigenvectors to reflect the structure characteristics and local scale
behavior of the vibration signal. Then, the principal component analysis (PCA) was introduced to
refine the eigenvectors for a higher recognition efficiency and accuracy. Finally, the vibration
signals of four types of reciprocating compressor valve faults were analyzed by this method, and
the faults were identified correctly by pattern classifiers of BTSVM and CNN. Further results
comparison with other feature extraction methods verifies the superiority of the proposed method. Keywords: fault diagnosis, reciprocating compressor valve, variational mode decomposition (VMD), multi-fractal detrended fluctuation analysis (MFDFA), principal component analysis (PCA).

\section{Introduction}

As general machinery, reciprocating compressor is widely used in petroleum and petrochemical industries, while due to its complex structure and many vulnerable parts, failures often occur and even lead to some severe consequences such as the production process termination and equipment destruction $[1,2]$. According to the statistics, valve is one of the most core and vulnerability components, and it accounts for $60 \%$ of the total failure rate of reciprocating compressors. Therefore, the valve fault diagnosis plays a significant role to guarantee the normal operating condition of reciprocating compressor. Vibration signals are widely used in the machinery fault diagnosis because they contain a lot of operating information. However, due to the nonlinearity, nonstationarity and multi-component coupling characteristics of reciprocating compressor valve vibration signals, an effective signal processing and information extraction methods are the key to the fault diagnosis process of a reciprocating compressor valve [3]. The traditional signal techniques, such as frequency-domain and time-domain statistical indicators, are not suitable for extracting the effective features from the vibration signals of reciprocating compressor valve faults [4].

As a novel tool for analyzing a mechanical fault signal, the Empirical Mode Decomposition (EMD), Local Mean Decomposition (LMD) and other self-adaptive signal processing methods proposed by the relevant scholars have been used widely in the field of mechanical vibration fault 
diagnosis [3-6]. Variational mode decomposition (VMD) is a new method of adaptive signal decomposition recently proposed by Dragomiretskiy and Zosso [7], which can decompose a complicated multi-component vibration signal into a set of modal functions with a limited bandwidth by solving the constrained variational model in the variational frame, and separate the frequency of each signal component. Compared with EMD and LMD, the VMD is a non-recursive processing method to solve the modal aliasing problem in the EMD which cannot correctly separate the components with similar frequencies due to the sampling frequency. Furthermore, the VMD is a number of adaptive Wiener filter banks, and thus it has more noise robustness and fast convergence [7-9]. In accordance with the advantages of VMD, it has been successfully utilized for extracting the fault features for nonlinear and non-stationary rub-impact signals [10], instantaneous detection of speech signals [8] and trends analysis of financial markets [11], etc. Therefore, VMD is an appropriate method to analyze reciprocating compressor valve vibration signals with the characteristics of nonlinearity and nonstationarity.

As an effective method for feature extraction methods, fractal analysis $[12,13]$ is utilized to reveal the non-stationarity and discontinuity characteristics hidden in signals, which can reflect dynamic mechanisms corresponding to the different statuses of non-linear systems. Further studies show that the multi-fractal analysis $[14,15]$ presents the fractal singular probability distribution form of the signal and improves the elaboration degree of signal geometric features and local scale behavior, and has been used for a multiple singularity analysis [16]. With the development of the multi-fractal theory, Kantelhardt et al. proposed a multi-fractal detrended fluctuation analysis (MFDFA) method [17]. Compared with conventional multi-fractal method, it can eliminate sequence trend terms by DFA, which fully reveal the multi-fractal features hidden in non-stationary time series, and estimate the multi-fractal spectrum accurately [18]. The literature studies $[16,19-22]$ show that the multi-fractal singularity spectrum obtained by the MFDFA is a set of parameters which can describe the dynamic behavior of the multi-fractal time series more accurately. However, the choice criterion of MFDFA parameters is still non-uniform in standards. Some scholars choose only one single singular exponent as the multi-fractal singularity spectrum parameter to form the eigenvector, while other scholars select the singular exponent $\alpha_{+\infty}, \alpha(0)$, $\alpha_{-\infty}, \Delta \alpha$ as the parameters of the multi-fractal singularity spectrum. Hence, the proper selection of parameters is very important to reflect the inherent information of multi-fractal spectrum and the dynamic behavior of time series. In this paper the multi-fractal singularity spectrum parameters are considered from two aspects, that is, singular exponent $\alpha$ and multi-fractal spectrum $f(\alpha)$, and it may realize the fault diagnosis of reciprocating compressor accurately.

Owing to the characteristics of nonlinearity, non-stationarity and multi-component coupling, the vibration signal of the reciprocating compressor contains interference noise. Therefore, it is necessary to pre-process the original signal for reducing or eliminating the noise disturbance before a further analysis. The proposed feature extraction method combined with the self-adaptive decomposition and fractal analysis has become a hot topic in the field of fault diagnosis. Jia, et al. [23] presented an intelligent diagnosis of bearing based on the EMD and MFDFA. Liu [24] proposed a feature extraction method based on the LCD-TEO and MFDFA for a rolling-bearing fault diagnosis. Therefore, to extract more accurate multi-fractal eigenvectors, a feature extraction method combined with VMD and MFDFA is proposed in this paper.

In this research, a novel feature extraction method based on the VMD and MFDFA is proposed, and its application for reciprocating compressor valve fault diagnosis is described. The remaining section of this paper is organized as follows: Section 2 introduces the principle of VMD and MFDFA. The method and procedures of feature extraction method are described in Section 3. The process of extracting features for different reciprocating compressor valve faults is presented in Section 4. Finally, the conclusion is outlined in Section 5. 


\section{VMD and MFDFA principles}

\subsection{VMD algorithm}

VMD is a newly developed methodology for adaptive time-frequency analysis signal decomposition based on Wiener filtering, Hilbert transform, analytic signal and frequency mixing, etc. The decomposition process is essentially a special iteration solving variational model, and can non-cursively decompose a multi-component signal into a series of band-limited intrinsic mode functions (BLIMFS) [7].

\subsubsection{Variation model establishment}

In order to establish a variational model of signal decomposition, the VMD defines the intrinsic mode function (IMF) as the amplitude modulation-frequency modulation (AM-FM), as follows:

$u_{k}(t)=A_{k}(t) \cos \left(\varphi_{k}(t)\right)$

where $A_{k}(t)$ is the instantaneous amplitude of $u_{k}(t)$ and $\varphi_{k}(t)$ is the instantaneous phase of $u_{k}(t), \varphi_{k}(t)$ is the reduction function that instantaneous frequency $\omega_{k}(t)=d \varphi_{k}(t) / d t \geq 0$. Compared to $\varphi_{k}(t)$, the variation of $A_{k}(t)$ and $\omega_{k}(t)$ is more gradual that can be regarded as a harmonic signal of constant amplitude and frequency in a smaller time horizon.

On the basis of this definition, the VMD assumes that the input signal $x(t)$ is composed of finite BLIMF components with different center frequencies and limited bandwidth, and transforms the signal decomposition problem into the variational model framework for processing. The minimum sum of the estimated bandwidths of each BLIMF component is sought under the constraint that the sum of the BLIMF components is equal to the input signal $x(t)$. The constraint variational model established by the VMD is as follows:

$$
\begin{aligned}
& \min _{\left\{u_{k}\right\},\left\{\omega_{k}\right\}}\left\{\sum_{k=1}^{K}\left\|\partial_{t}\left[\left(\delta(t)+\frac{j}{\pi t}\right) * u_{k}(t) e^{-j \omega_{k} t}\right]\right\|_{2}^{2}\right\}, \\
& \text { subject to } \sum_{k=1}^{K} u_{k}(t)=f(t),
\end{aligned}
$$

where $K$ is the number of the BLIMF component, $\left\{u_{k}\right\}=\left\{u_{1}, u_{2}, \ldots, u_{K}\right\}$ and $\left\{\omega_{k}\right\}=\left\{\omega_{1}, \omega_{2}, \ldots, \omega_{K}\right\}$ are frequency centers of $u_{k}(t) . \delta(t)$ is Dirichlet function, $*$ symbol is convolution symbol.

\subsubsection{Variation model solution}

In order to obtain the optimal solution of the above variational model, the VMD constructs the extended Lagrangian function $L\left(\left\{u_{k}\right\},\left\{\omega_{k}\right\}, \lambda\right)$ by introducing a quadratic penalty factor $\alpha$ and Lagrangian multiplier $\lambda(t)$, as shown in Eq. (3), the constraint problem is transformed into an unconstrained problem. Among them, the quadratic penalty factor can ensure the accuracy of signal reconstruction in the presence of Gaussian noise, while Lagrangian multiplier can guarantee the rigidity of the model constraints: 


$$
\begin{aligned}
& L\left(\left\{u_{k}\right\},\left\{\omega_{k}\right\}, \lambda\right)=\sum_{k=1}^{K}\left\|\partial_{t}\left[\left(\delta(t)+\frac{j}{\pi t}\right) * u_{k}(t)\right] r^{-j \omega_{k} t}\right\|_{2}^{2}+\left\|x(t)-\sum_{k=1}^{K} u_{k(t)}\right\|_{2}^{2} \\
& +\left\langle\lambda(t), x(t)-\sum_{k=1}^{K} u_{k}(t)\right\rangle .
\end{aligned}
$$

On the basis of this, the alternating direction method of multipliers (ADMM) is used to update $\left\{u_{k}\right\},\left\{\omega_{k}\right\}$ and $\lambda$, the saddle point of the extended Lagrangian function is sought to be the optimal value of the variational model shown in Eq. (2), thus decomposing the input signal $x(t)$ into $K$ BLIMF components. The VMD algorithm is a specific process shown in Fig. 1.

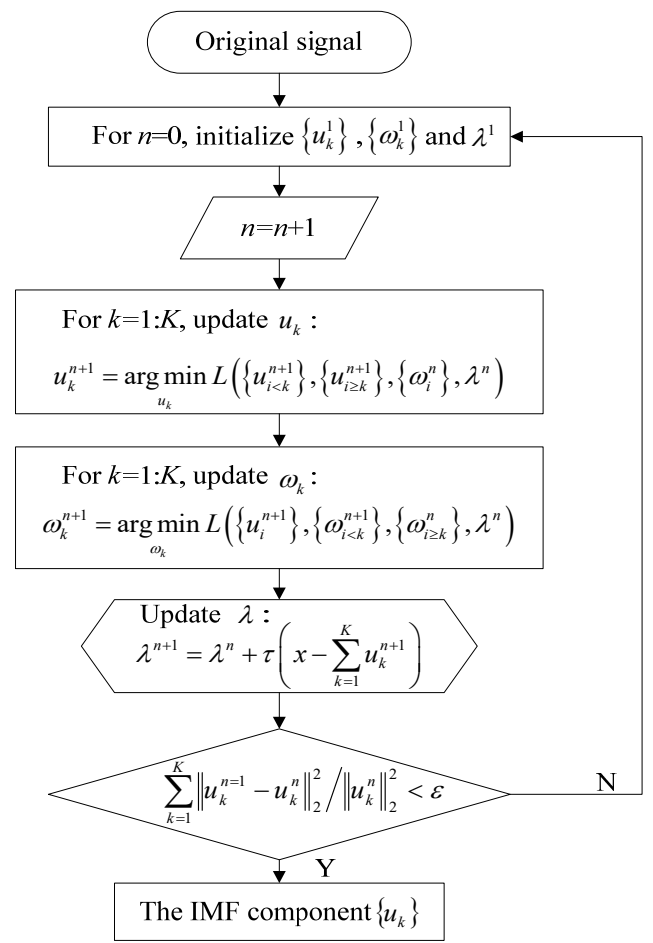

Fig. 1. Flow chart of VMD algorithm

\subsection{MFDFA}

\subsubsection{Method description}

For non-stationary time series $x_{k}$, the process of MFDFA is as follows:

$Y(i)=\sum_{k=1}^{i}\left(x_{k}-\langle x\rangle\right), i=1,2, \cdots, N$,

1) Construction of Signal Outlines

where $\langle x\rangle$ is the mean value of time series $x_{k}$.

Divide $Y(i)$ into $N_{s}=\operatorname{int}(N / s)$ non-overlapping segments of equal length s. To take the full advantage of this series, repeat the same process from the opposite end. Consequently, $2 N_{s}=\operatorname{int}(N / s)$ segments are obtained altogether. 
The least square method is used to fit the polynomial trend of $2 N_{s}=\operatorname{int}(N / s)$ segments, and then to calculate the variance:

$F^{2}(v, s)=\sum_{i=1}^{s}\left\{Y[(v-1) s+i]-y_{v}(i)\right\}^{2}(v=1,2, \cdots, N)$,
$F^{2}(v, s)=\sum_{i=1}^{s}\left\{Y\left[N-\left(v-N_{S}\right) s+i\right]-y_{v}(i)\right\}^{2} \quad\left(v=N_{S}+1, N_{S}+2, \cdots, 2 N_{S}\right)$,

where $Y$ is the trend of the polynomial data of the $v$ th segment. If the fitted polynomial trend is order $m$, then the trend process is (MF) $\mathrm{DFA}_{\mathrm{m}}$.

Calculate the mean value of the $q$ th order fluctuation function:

$F_{q}(s)=\left\{\frac{1}{2 N_{s}} \sum_{v=1}^{2 N_{s}}\left[F^{2}(v, \quad s)\right]^{q / 2}\right\}^{1 / q}$,

where $q$ is non-zero real numbers.

4) If there is self-similarity of the time series $x_{k}$, there is a power-law relationship between the mean $F q(s)$ of the $q$ th fluctuation function and time scale $s$ :

$F_{q}(s) \propto s^{h(q)}$,

where $h(q)$ is the generalized Hurst exponent. If $x_{k}$ is a multi-fractal time series, then the exponent $h(q)$ will vary with $q$; if $x_{k}$ is a single multi-fractal time series, then the exponent $h(q)$ is a constant independent of $q$.

Analytically the relation between the generalized Hurst exponent $h(q)$ obtained by Eq. (8) and the scaling exponent $\tau(q)$ obtained by the standard partition function as follows:

$\tau(q)=q h(q)-1$

The multi-fractal singular exponent $\alpha$ and multi-fractal spectrum $f(\alpha)$ can be obtained by the Legendre transform:

$\alpha=\frac{d \tau(q)}{d q}=h(q)+q h^{\prime}(q)$

$f(\alpha)=q \alpha-\tau(q)=q[\alpha-h(q)]+1$.

\subsection{MFDFA parameters}

The multi-fractal singular spectrum obtained by MFDFA method is a set of parameters which can describe the dynamic behavior of the multi-fractal time series. The singular exponent $\alpha$ reflects the growth probability of the fractal in a small area. The singular spectrum $f(\alpha)$ is the fractal distribution function of the singular exponent $\alpha$. The multi-fractal singular spectrum $f(\alpha)$ has three characteristic points, namely the left and right end points and the extreme point. As a typical multi-fractal singular spectrum shown in Fig. 2, the MFDFA parameters are the left end point $\left(\alpha_{+\infty}, f\left(\alpha_{+\infty}\right)\right)$, right end point $\left(\alpha_{-\infty}, f\left(\alpha_{-\infty}\right)\right)$, the width of the multi-fractal singular spectrum $\Delta \alpha=\alpha_{-\infty}-\alpha_{+\infty}$, and the degree of inhomogeneity $\alpha_{0}$ in the extreme point.

To evaluate the above proposed multi-fractal singular spectrum parameters, the multi-fractal singular spectrum of a normal state and valve plate fracture state vibration signals were given in Fig. 3, and the multi-fractal singular spectrum parameters were listed in Table 1. From Fig. 3 and 
Table 1, we can see that shape and position coordinates of multi-fractal singular spectrum are different for two fault states, because the shape of the multi-fractal singular spectrum reflects the characteristics of the vibration signal probability distribution apparently. Based on the above discussion, the six-dimensional eigenvector $\left(\alpha_{+\infty}, f\left(\alpha_{+\infty}\right), \alpha_{0}, \alpha_{-\infty}, f\left(\alpha_{-\infty}\right), \Delta \alpha\right)$ composed of three feature points of the multi-fractal singular spectrum can reveal the inherent dynamics of non-stationary time series, so they are suitable as the characteristic parameters of the vibration signal.

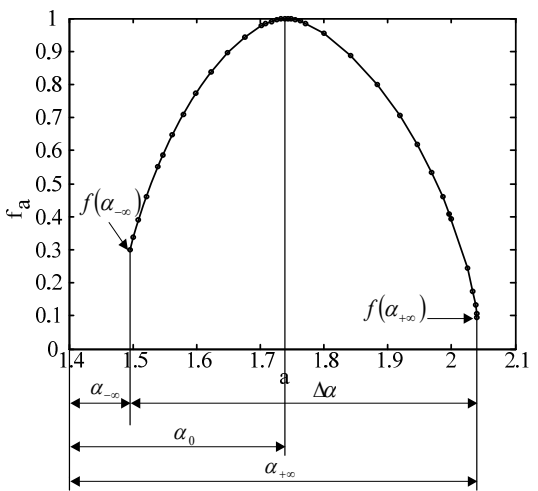

Fig. 2. Characteristic parameters of multi-fractal singular spectrum

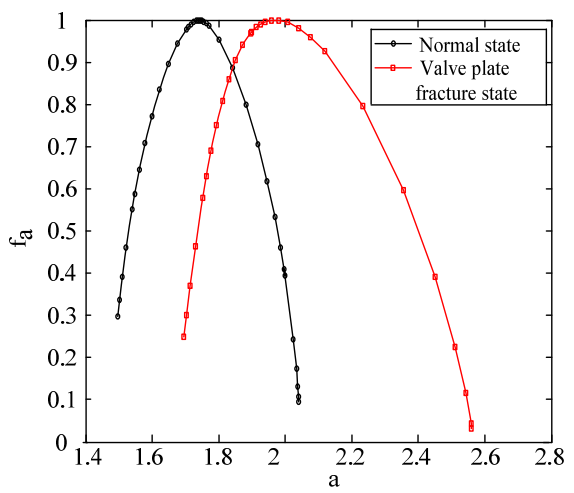

Fig. 3. Multi-fractal singular spectrum of cycle of normal state and valve fracture state vibration signals

Table 1. Comparison of multi-fractal singular spectrum parameters

\begin{tabular}{|c|c|c|c|c|c|c|}
\hline \multirow{2}{*}{ Valve states } & \multicolumn{5}{|c|}{ Multi-fractal singular spectrum parameters } \\
\cline { 2 - 7 } & $\alpha_{+\infty}$ & $f\left(\alpha_{+\infty}\right)$ & $\alpha_{0}$ & $\alpha_{-\infty}$ & $f\left(\alpha_{-\infty}\right)$ & $\Delta \alpha$ \\
\hline Normal state & 2.0401 & 0.0937 & 1.7438 & 1.4955 & 0.2983 & 0.5447 \\
\hline Valve plate fracture state & 2.5587 & 0.2487 & 1.9591 & 1.6961 & 0.0303 & 0.8626 \\
\hline
\end{tabular}

\section{Feature extraction method based on VMD and MFDFA}

The VMD can self-adaptively decompose a complicated multi-component signal into a series of BLIMFs which contain information about the specific frequency band of the original vibration signal, and the MFDFA is used to eliminate the influence of non-stationary trend of time series through a de-trend process. In this section, a feature extraction method based on VMD and MFDFA is proposed.

The VMD can decompose each fault state vibration signal into a series of BLIMF components with a given $K$ value, and the $K$ value may vary for different fault states. Therefore, it is necessary to select a uniform parameter $K$ of VMD decomposition for all fault states.

To solve this problem, the parameter $K$ in VMD is chosen adaptively by using the Minimum Redundancy Maximum Relevance (MRMR). The MRMR criterion is essentially a feature selection method, and its core idea is to use mutual information to calculate the correlation and the redundancy between feature parameters and classification targets. Theoretically, the ideal signal decomposition result should be that each BLIMF component has a large correlation with the original signal, so that the original signal characteristic is well inherited.

Assuming VMD to decompose the vibration signal of the reciprocating compressor valve $x(t)$ to obtain $K$ number BLIMF components $\left\{u_{k}\right\}=\left\{u_{1}, u_{2}, \cdots, u_{K}\right\}$, the correlation $D$ of $K$ number BLIMF components can be expressed as:

$D=\frac{1}{K} \sum_{u_{i} \in\left\{u_{k}\right\}} I\left(u_{i}, x\right)$, 
where $I\left(u_{i}, x\right)$ represents the mutual information between $u_{i}(t)$ and $x(t)$.

Similarly, the redundancy $R$ of the $K$ number BLIMF components can be expressed as:

$R=\frac{2}{K(K-1)} \sum_{u_{i}, u_{j} \in\left\{u_{k}\right\}, i \neq j} I\left(u_{i}, u_{j}\right)$.

In summary, the MRMR criterion is obtained as follows [13]:

$\max (M), M=D-\beta R$,

where $M$ is the MRMR criterion function and $\beta$ is the adjustment factor. The correlation between the BLIMF component, and the original signal is more important than the redundancy, so in this paper $\beta$ is chosen to 0.6 .

The MFDFA can analyze each BLIMF component and obtain six multiple $K$ cells for one fault state eigenvector feature vectors. However, for the diagnosis of the reciprocating compressor valve, the enormous amount of eigenvectors may reduce its robustness, and even lead to a decline in the diagnostic accuracy. Therefore, the eigenvector needs to be reduced to extract highlighted information. The Principal Component Analysis (PCA) is an effective method to extract the feature of the high-dimensional data [25]. It can be used to replace the original variables by constructing a new comprehensive variable, which can effectively reflect the integrated information of the original variable.

Based on the above discussion, the scheme of the fault feature extraction method is shown in Fig. 4, and the scheme of the method is briefly described as follows:

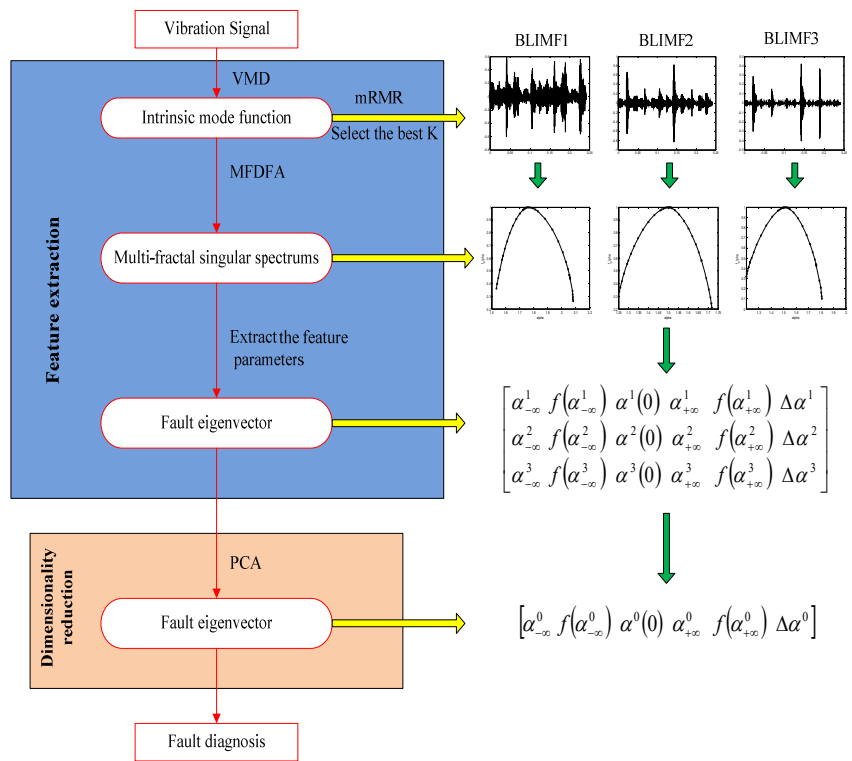

Fig. 4. Fault diagnosis scheme

1) For the vibration signals of different fault states, we employ the VMD method to decompose each signal into $K$ ( $K$ are usually from 2 to 8 ) number of BLIMFs, and repeat the decomposition VMD method with another $K$ ( $K$ are usually from 2 to 8 );

2) For the BLIMFs decomposed by VMD using one number $K, M$ values are calculated for BLIMFs of each fault state respectively, and the $M$ values are added in each fault state together, then this calculation is repeated for all parameters $K$ (usually from 2 to 8 ), and the best $K$ number is selected from BLIMFs which has the biggest sum of $M$ values for all fault states; 
3) For $K$ (a special value has been selected) number of BLIMFs, the multi-fractal spectrum of each BLIMF component is calculated in one fault state, then the left end point $\left(\alpha_{-\infty}, f\left(\alpha_{-\infty}\right)\right)$, right end point $\left(\alpha_{+\infty}, f\left(\alpha_{+\infty}\right)\right)$, extreme point $\alpha(0)$, and the width of multi-fractal singular spectrum $\Delta \alpha$ are chosen to construct the six-dimensional eigenvector $\left(\alpha_{+\infty}, f\left(\alpha_{+\infty}\right), \alpha(0)\right.$, $\left.\alpha_{-\infty}, f\left(\alpha_{-\infty}\right), \Delta \alpha\right)$, and there are six multiple $K$ cells for one fault state eigenvector;

4) The huge amount of cells in eigenvector will decrease the diagnosis accuracy, therefore, the PCA is utilized to reduce the dimension of previously extracted eigenvector, then the PCA dimension reduced eigenvector is taken as the final eigenvector.

\section{Fault diagnosis for reciprocating compressor valve based on VMD and MFDFA method}

In this study, the proposed VMD and MFDFA method is utilized to extract the fault feature of the valve vibration data from a two-stage double-acting reciprocating compressor of type 2D12 (As shown in Fig. 5). The shaft power of reciprocating compressor is $500 \mathrm{~kW}$, the piston stroke is $240 \mathrm{~mm}$, and the motor speed is $496 \mathrm{rpm}$.
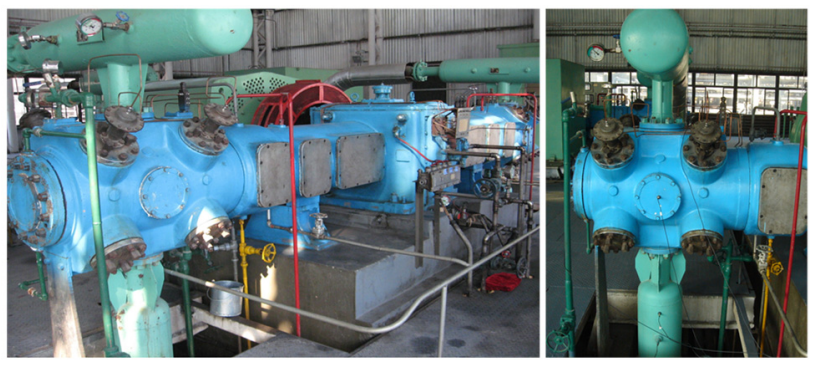

a) Original figure of reciprocating compressor

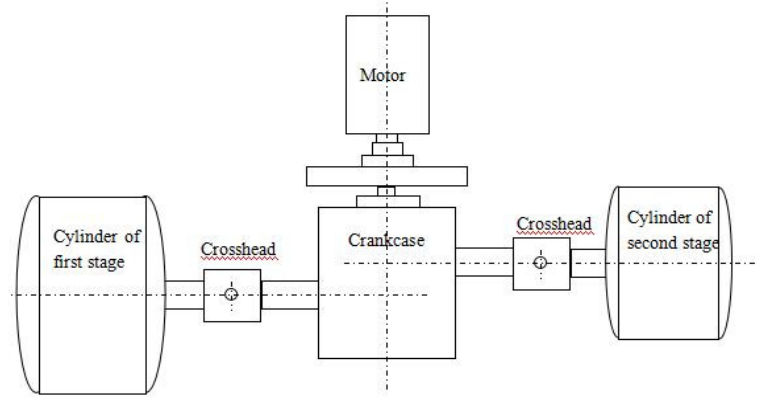

b) Structural drawing of reciprocating compressor

Fig. 5. Two-stage double-acting reciprocating compressor of 2D12 type

Valve is one of the core components of reciprocating compressor, its safe and stable operation is of great significance for the reciprocating compressor. The 2D12 reciprocating compressor valve is a ring valve which is composed of valve seat, valve plate, valve lift limiter, spring, screw and nut. Due to the long-term effect of the alternating load, the valve with periodic reciprocating motion is more prone to a failure. This paper mainly investigates three kinds of reciprocating compressor valve failures: spring failure, valve plate fracture and valve plate gap. When the reciprocating compressor valve is abnormal, the performance of the vibration signal in the valve cover side direction will undergo a substantial change. Therefore, this paper extracted valve cover vibration signals as the analysis data. The vibration signals of four primary valve states are shown in Fig. 6 for two periods.

In this paper, we employed the proposed VMD and MFDFA method to extract features for reciprocating compressor valves faults. Using the VMD to decompose the vibration signal of the reciprocating compressor valve faults, the key point is to select the number of parameters $K$. We 
used the MRMR to choose the appropriate parameter $K$ for the vibration signals of reciprocating compressor valves.
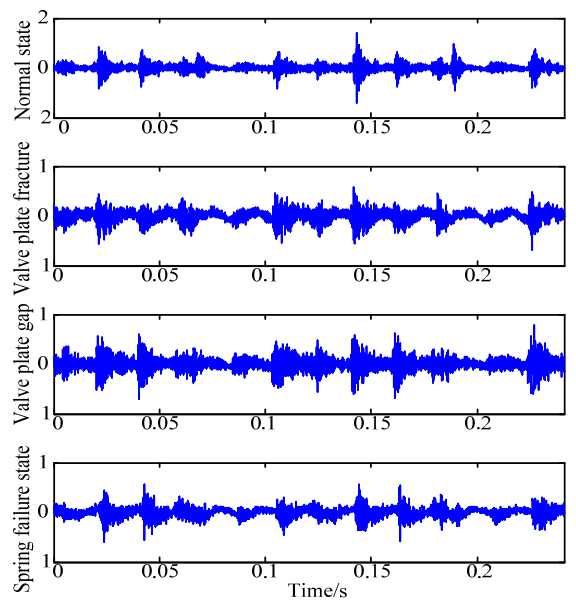

Fig. 6. Vibration acceleration in four reciprocating compressor valve states

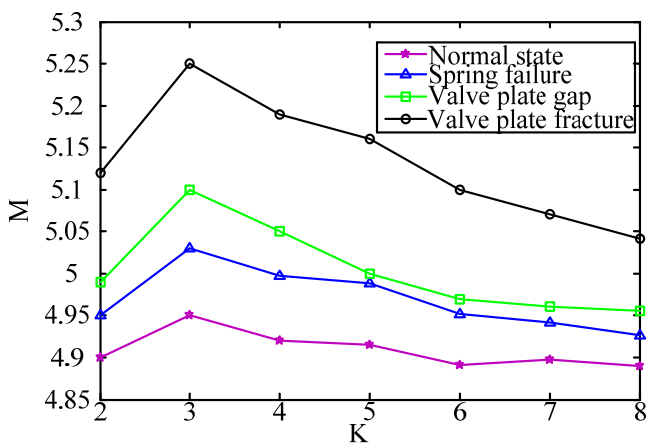

Fig. 7. MRMR criterion function $M$ values

Firstly, four different vibration signals of valve faults were decomposed by the VMD with $K=2,3, \ldots, 8$, respectively. Then, according to Eq. (12) to (14) $M$ values of MRMR criterion function are calculated for BLIMFs in each fault state with a special $K$ value. The $M$ values for each fault state with different $K$ are shown in Fig. 7. It can be seen from Fig. 7 that the $M$ values tend to increase first and then to decrease with the parameter $K$. When $K=3$, the sum of $M$ values for four different states reach the maximum. Therefore, in the following study, $K=3$ is chosen to decompose the valve fault signals.

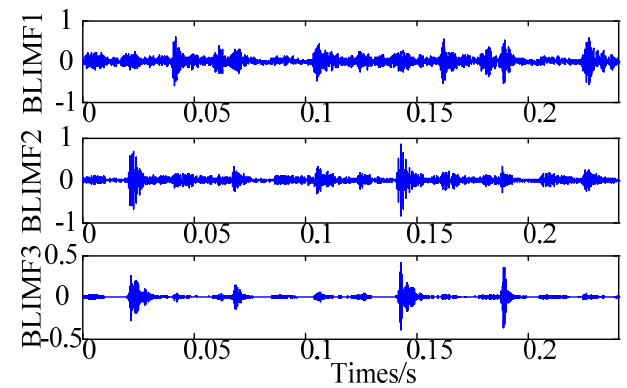

Fig. 8. BLIMFs of vibration acceleration in normal state

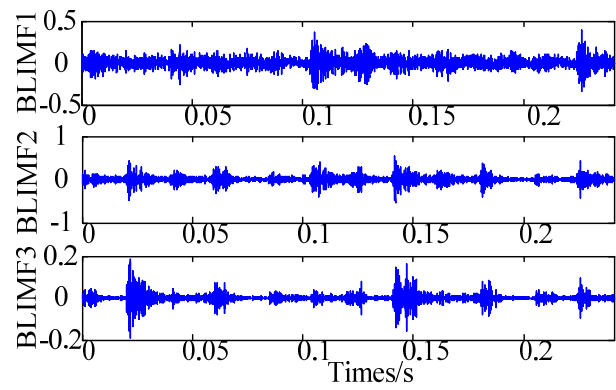

Fig. 9. BLIMFs of vibration acceleration in valve plate fracture state 


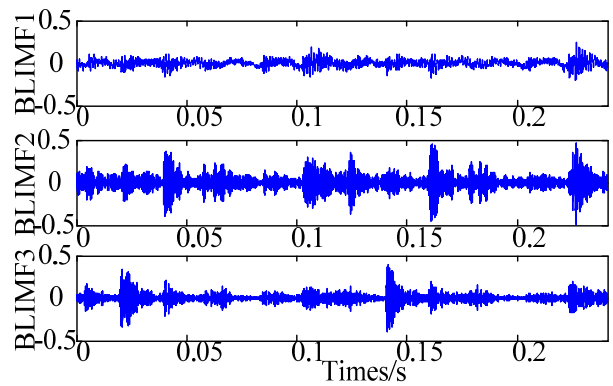

Fig. 10. BLIMFs of vibration acceleration in valve plate gap state

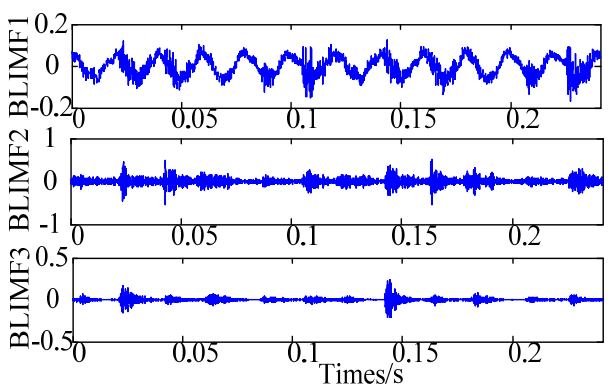

Fig. 11. BLIMFs of vibration acceleration in spring failure state

The vibration signals of four valve fault states were decomposed into three BLIMF components by VMD, and they were shown in Figs. 8-11 respectively. Afterwards, three BLIMF components in each fault state were calculated by the MFDFA to obtain the multi-fractal singular spectrums. In this paper, the scaling exponent $q$ in MFDFA was set as -10 to 10. In order to show the differences, the multi-fractal singular spectrum of each BLIMF component for four fault states as shown in Figs. 12-14 respectively.

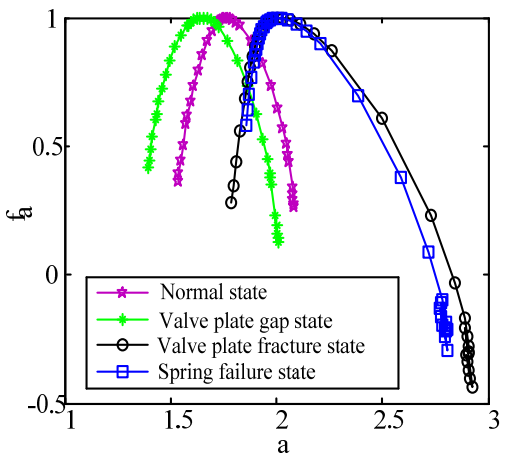

Fig. 12. Multi-fractal singular spectrums of BLIMF1 components in all states

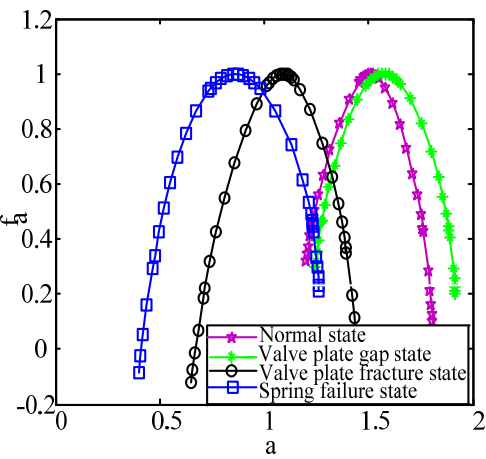

Fig. 13. Multi-fractal singular spectrums of BLIMF2 components in all states

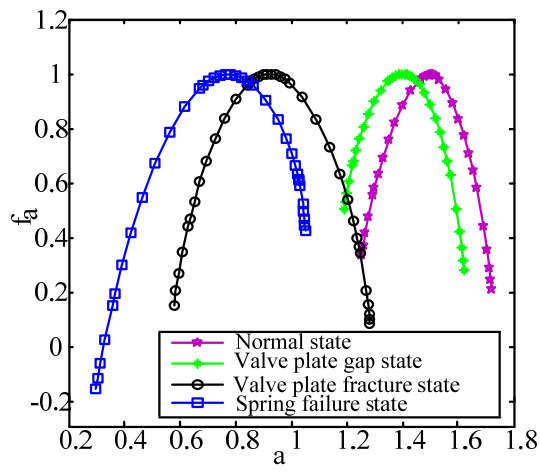

Fig. 14. Multi-fractal singular spectrums in of BLIMF3 components in all states

It can be seen that the multi-fractal singular spectrum of each state is a parabola with downward opening, and the instantaneous amplitude of the multi-fractal singular spectrums under four fault states are distinguished from each other, and the position, span and size of the multi-fractal singular spectrum are also different. The multi-fractal singular spectrum index $\alpha$ changes within a large range, and demonstrates that the vibration signal is a multi-fractal process. Besides, the 
value of $\Delta \alpha, \Delta f$ under normal condition is the smallest, while those of fault states are obviously higher than the normal state. In brief, the six-dimensional eigenvector $\left(\alpha_{+\infty}, f\left(\alpha_{+\infty}\right), \alpha(0), \alpha_{-\infty}\right.$, $\left.f\left(\alpha_{-\infty}\right), \Delta \alpha\right)$ of the multi-fractal singular spectrum, is able to reveal the differences of multi-fractal characteristics.

In Table 2, we listed the six-dimensional eigenvector of three BLIMF components for each fault state in Figs. 12-14, and the numerical values of eigenvectors have a significant difference between four valve states. Then we utilize the PCA to extract the highlighted information, and the final fault eigenvector was shown in Table 3. The cells of eigenvector in Table 2 were reduced from 18 to 6 for each fault state, and it contributes for a higher recognition efficiency and accuracy.

Table 2. Multi-fractal parameters of each BLIMF component in different valve states

\begin{tabular}{|c|l|c|c|c|c|c|c|}
\hline \multicolumn{2}{|c|}{ Valve states } & \multicolumn{7}{|c|}{ Multi-fractal parameters } \\
\cline { 2 - 8 } & & $\alpha_{+\infty}$ & $f\left(\alpha_{+\infty}\right)$ & $\alpha(0)$ & $\alpha_{-\infty}$ & $f\left(\alpha_{-\infty}\right)$ & $\Delta \alpha$ \\
\hline \multirow{3}{*}{ Normal state } & BLIMF1 & 1.8559 & 0.1644 & 1.5740 & 1.3428 & 0.3884 & 0.5131 \\
\cline { 2 - 8 } & BLIMF2 & 1.8922 & 0.0797 & 1.5815 & 1.2689 & 0.2040 & 0.6233 \\
\cline { 2 - 8 } Valve plate fracture & BLIMF3 & 1.8234 & 0.2714 & 1.5503 & 1.2102 & 0.4092 & 0.6133 \\
\hline \multirow{3}{*}{ Valve plate gap } & BLIMF1 & 2.5227 & -0.0992 & 1.9543 & 1.6839 & 0.0954 & 0.8388 \\
\cline { 2 - 8 } & BLIMF2 & 1.6836 & -0.0979 & 1.3301 & 0.9827 & 0.1959 & 0.7009 \\
\cline { 2 - 8 } & BLIMF3 & 1.8544 & -0.2050 & 1.3104 & 0.8019 & -0.0101 & 1.0525 \\
\hline \multirow{3}{*}{ Spring failure } & BLIMF1 & 1.9940 & 0.0008 & 1.5264 & 1.2443 & 0.3788 & 0.7497 \\
\cline { 2 - 8 } & BLIMF2 & 1.7502 & 0.0980 & 1.3867 & 1.1139 & 0.5932 & 0.6362 \\
\cline { 2 - 8 } & BLIMF3 & 1.8354 & 0.1800 & 1.5057 & 1.1384 & 0.3682 & 0.6970 \\
\hline & BLIMF1 & 2.8369 & -0.3271 & 2.0045 & 1.8578 & 0.6212 & 0.9791 \\
\cline { 2 - 8 } & BLIMF2 & 1.0954 & -0.1606 & 0.5830 & 0.2464 & 0.1829 & 0.8490 \\
\cline { 2 - 8 } & BLIMF3 & 1.0827 & 0.1442 & 0.6979 & 0.1635 & 0.0619 & 0.9192 \\
\hline
\end{tabular}

Table 3. Final state eigenvector in different valve states

\begin{tabular}{|c|c|c|c|c|c|c|}
\hline Valve states & \multicolumn{7}{|c|}{ Final state eigenvector } \\
\hline Normal & -0.0357 & -0.1070 & 0.1650 & 0.0340 & -0.1293 & 0.0730 \\
\hline Valve plate fracture & -0.8435 & 0.0079 & 0.4011 & -0.2479 & 0.4423 & 0.2400 \\
\hline Valve plate gap & 0.1960 & 0.0694 & -0.2091 & 0.0571 & 0.0131 & -0.1265 \\
\hline Spring failure & 1.8864 & 0.0035 & -0.9158 & -0.1818 & -0.9707 & 0.1783 \\
\hline
\end{tabular}

Further evaluation is needed to assess the effectiveness of the proposed method based on the VMD and MFDFA, and this paper introduces the Binary Tree Support Vector Machine (BTSVM) and Convolutional Neural Network (CNN) methods to evaluate four valve fault eigenvectors. To test this, 150 eigenvector samples were selected from each valve fault respectively, and 100 were taken as training samples, other 50 as test samples. For the BTSVM, the radial basis kernel function was employed, and the kernel parameter $\gamma=3.57$ and error penalty parameter $C=1.85$ were optimized by the genetic algorithm. The $\mathrm{CNN}$ is made up of an input layer, convolution layer, sampling layer, full connection layer and output layer [26, 27]. The size and number of convolution kernel and the sampling width of CNN have an important influence on the fault classification results. Therefore, according to the study of the eigenvector data, the convolution kernel size is set as $2 \times 1$, the number of convolution kernel is as 8 , the sampling width is as $2 \times 1$, the number of neurons in the full connection layer as 8 is based on the experience and constant experimentation. Meanwhile, the weights and bias of the model network are initialized to a random number between 0 and 1. For each fault state, 50 samples were recognized by BTSVM and CNN respectively, and the results are shown in Table 4.

To compare the superiority of this feature extraction method, the same number of training and test samples was extracted by other four methods including EMD-MFDFA and PCA, Wavelet packet-MFDFA and PCA, MFDFA and PCA, and VMD-MFDFA, and then recognized using the BTSVM and CNN. The recognition results are also listed in Table 4. 
Table 4. Recognition accuracy of comparison between different methods

\begin{tabular}{|c|c|c|c|c|c|c|c|c|c|c|}
\hline \multirow{2}{*}{$\begin{array}{c}\text { Feature } \\
\text { extraction } \\
\text { method }\end{array}$} & \multicolumn{6}{|c|}{ Normal } & \multicolumn{2}{|c|}{$\begin{array}{c}\text { Spring } \\
\text { failure }\end{array}$} & $\begin{array}{c}\text { Valve plate } \\
\text { fracture }\end{array}$ & \multicolumn{2}{c|}{$\begin{array}{c}\text { Valve plate } \\
\text { gap }\end{array}$} & \multicolumn{2}{|c|}{$\begin{array}{c}\text { Total accuracy } \\
(\%)\end{array}$} \\
\cline { 2 - 12 } & BT & CNM & $\begin{array}{c}\text { BT } \\
\text { SVM }\end{array}$ & CNN & $\begin{array}{c}\text { BT } \\
\text { SVM }\end{array}$ & CNN & $\begin{array}{c}\text { BT } \\
\text { SVM }\end{array}$ & CNN & $\begin{array}{c}\text { BT } \\
\text { SVM }\end{array}$ & CNN \\
\hline $\begin{array}{c}\text { VMD-MFDA } \\
\text { and PCA }\end{array}$ & 98 & 100 & 96 & 98 & 100 & 98 & 98 & 100 & 98 & 99 \\
\hline $\begin{array}{c}\text { EMD-MFDFA } \\
\text { and PCA }\end{array}$ & 96 & 98 & 92 & 94 & 96 & 96 & 90 & 92 & 93.5 & 95 \\
\hline $\begin{array}{c}\text { Wavelet packet- } \\
\text { MFDFA and } \\
\text { PCA }\end{array}$ & 92 & 92 & 90 & 92 & 92 & 94 & 90 & 90 & 91 & 92 \\
\hline $\begin{array}{c}\text { MFDFA and } \\
\text { PCA }\end{array}$ & 90 & 90 & 88 & 90 & 90 & 92 & 90 & 92 & 89.5 & 91 \\
\hline VMD-MFDFA & 94 & 94 & 90 & 92 & 94 & 96 & 90 & 90 & 92 & 93 \\
\hline
\end{tabular}

The results show that both the accuracy in each state and the total accuracy of the proposed method are better than those of four other feature extraction methods under the condition of the same finite number of samples. Feature vectors extracted by VMD-MFDFA and PCA, the EMD-MFDFA and PCA and Wavelet packet-MFDFA and PCA are better than those achieved by the MFDFA and PCA from the result of recognition rate, so it is necessary to combine the self-decomposition method with MFDFA for resisting noise interference and highlighted information extraction, while the VMD is more superior method than the EMD and Wavelet packet for these fault vibration signals according to the better recognition rate. From Table 4, we also see that the fault recognition rates of VMD-MFDFA without PCA are lower than those of the proposed method, so it validates the necessity of using PCA in this method. Through the comparative researches above, the proposed method is the superior feature extraction method to diagnosis faults of reciprocating compressor valve effectively and accurately.

\section{Conclusions}

According to the nonlinearity, nonstationarity and multi-component coupling characteristics of reciprocating compressor vibration signals, this paper presents a novel feature extraction method based on the VMD and MFDFA, and it is applied for the fault diagnosis of reciprocating compressor valve faults.

1) In the proposed method, a novel VMD method was employed to eliminate the noise interference, which outperforms the traditional time frequency analysis method such as the EMD and Wavelet packet for the reciprocating compressor vibration signals.

2) After the BLIMF components being characterized by the MFDFA method, the PCA was employed to refine the final eigenvector, and it indeed has a higher recognition efficiency and accuracy.

3) This method was applied for the fault diagnosis of reciprocating compressor at different valve states, and it demonstrates superior recognition results through the comparison with other feature extraction methods.

\section{Acknowledgements}

This work was partly supported by the General Financial Grant from the China Postdoctoral Science Foundation (2015M581423), School Cultivate Fund of Northeast Petroleum University in China (XN2014105), Natural Science Foundation in China (51505079), Natural Science Foundation of Heilongjiang Province in China (E2015037, E2016009), Supported by the Northeast Petroleum University Innovation Foundation For Postgraduates 
(YJSCX2017-020NEPU) and Basic Science and Engineering on Special Project of Heilongjiang Province Education Department (135109219).

\section{References}

[1] Elhaj M., Gub F., Ballb A. D. Numerical simulation and experimental study of two-stage reciprocating compressor for condition monitoring. Mechanical Systems and Signal Processing, Vol. 22, 2008, p. 374-389.

[2] Almasi A. New study and model for the mechanism of process reciprocating compressors and pumps. Proceedings of the Institution of Mechanical Engineers, Part E: Journal of Process Mechanical Engineering, Vol. 224, 2010, p. 143-148.

[3] Zhang S., Xu M., Li Y., Zhao H., Wang R. Valve fault diagnosis of reciprocating compressor based on hybrid method. Journal of Vibration and Shock, Vol. 35, 2016, p. 167-173.

[4] Zhao H., Wang J., Jie X., Gao Y. Feature extraction method based on LMD and MSE and its application for fault diagnosis of reciprocating compressor. Journal of Vibroengineering, Vol. 17, Issue 7, 2015, p. 3515-3526.

[5] Tang Y., Liu Q. Y., Xie C., et al. Study on stress distribution of a subsea Ram BOP body based on simulation and experiment. Engineering Failure Analysis, Vol. 50, 2015, p. 39-50.

[6] Wang J. D., Gao Y. Q., Zhao H. Y., Cong R. Sectional normalization and recognization on the PV-Diagram of reciprocating compressor. Journal of Measurements in Engineering, Vol. 3, 2015, p. 35-41.

[7] Dragomiretskiy K., Zosso D. Variational mode decomposition. IEEE Transactions on Signal Process, Vol. 62, Issue 3, 2014, p. 531-44.

[8] Upadhyay A., Pachori R. B. Instantaneous voiced/non-voiced detection in speech signals based on variational mode decomposition. Journal of The Franklin Institute, Vol. 352, 2015, p. 2679-2707.

[9] Aneesh C., Kumar S., Hisham P. M., Soman K. P. Performance comparison of variational mode decomposition over empirical wavelet transform for classification of power quality disturbances using support vector machine. Procedia Computer Science, Vol. 46, 2015, p. 372-380.

[10] Wang Y., Markert R., Xiang J., Zheng W. Research on variational mode decomposition and its application in detecting rub-impact fault of rotor system. Mechanical Systems and Signal Processing, Vol. 60, 2015, p. 243-51.

[11] Lahmiri S. Long memory in international financial markets trends and short movements during 2008 financial crisis based on variational mode decomposition and detrended fluctuation analysis. Physica A, Vol. 437, 2015, p. 130-138.

[12] Li P., He Q., Kong F. Approach to fault diagnosis of bearings using wavelet-based fractal analysis. Proceedings of the IEEE International Conference on Information and Automation (ICIA), 2010, p. 2338-2343.

[13] Wang Q., Zhang X. Application of fractal theory to fault diagnosis for hydraulic pump. Journal of Dalian Maritime University Vol. 2, 2004, p. 012.

[14] Yu Y., Baoliang L., Jingshan S., Shixuan Y. Application of vibration signal multi-fractal in fault diagnosis. Proceedings of the IEEE 2nd International Conference on Future Networks, 2010, p. 164-167.

[15] Ke P. Z., Yong H. Y., Qing L., Fulei C. Wavelet multifractal spectrum: application to analysis vibration signals. Chinese Journal of Mechanical Engineering. Vol. 8, 2002, p. 13.

[16] Wang J., Wei W., Li H., Li X. Multifractal features extraction of bearing faults for reciprocating compressor. Journal of Vibration and Shock, Vol. 27, 2008, p. 313-315.

[17] Kantelhardt J. W., Zschiegner S. A., Koscielny-Bunde E., Havlin S., Bunde A., Stanley H. E. Multifractal detrended fluctuation analysis of non-stationary time series. Physica A: Statistical Mechanics and its Applications, Vol. 316, 2002, p. 87-114.

[18] Talebinejad M., Chan A. D. C., Miri A. Fatigue estimation using novel multi-fractal detrended fluctuation analysis-based approach. Journal of Electromyography and Kinesiology, Vol. 20, Issue 3, 2010, p. 433-439.

[19] Li M., Ma W. X., Liu X. J. Investigation of rolling bearing fault diagnosis based on multi-fractal and general fractal dimension. 2nd Intelligent Computation Technology and Automation, IEEE Computer Society, Zhangjiajie, 2009, p. 545-548.

[20] Yu Y., Li B. L., Shang J. S., et al. Application of vibration signal multi-fractal in fault diagnosis. 2nd International Conference on Future Networks, IEEE Computer Society, Sanya, 2010, p. 164-167. 
[21] Tang J. Y., Shi Y. B., Zhou L. F., et al. Nonlinear analog circuit fault diagnosis using wavelet leaders multifractal analysis method. Control and Decision, Vol. 25, Issue 4, 2010, p. 605-609.

[22] Lin Jinshan, Chen Qian Fault diagnosis of rolling bearings based on multifractal detrended fluctuation analysis and Mahalanobis distance criterion. Mechanical Systems and Signal Processing, Vol. 38, Issue 2, 2013, p. 515-533.

[23] Jia F., Wu B., Xiong X. Y., Xiong S. B. Intelligent diagnosis of bearing based on EMD and multifractal detrended fluctuation analysis. Journal of Central South University (Science and Technology), Vol. 46, Issue 2, 2015, p. 491-497.

[24] Liu H. G., Wang X., Lu C. Rolling bearing fault diagnosis based on LCD-TEO and multifractal detrended fluctuation analysis. Mechanical Systems and Signal Processing. Vols. 60-61, 2015, p. 273-288.

[25] Dunia R., Qin Joe S. Joint diagnosis of process and sensor faults using principal component analysis, Control Engineering Practice, Vol. 6, 1998, p. 457-469.

[26] Karpathy A., Toderici G., Shetty S., et al. Large-scale video classification with convolutional neural networks. IEEE Conference on Computer Vision and Pattern Recognition, 2014, p. 1725-1732.

[27] Oquab M., Bottou L., Laptev I., et al. Learning and transferring mid-level image representations using convolutional neural networks. IEEE Computer Vision and Pattern Recognition. 2014, p. $1717-1724$.
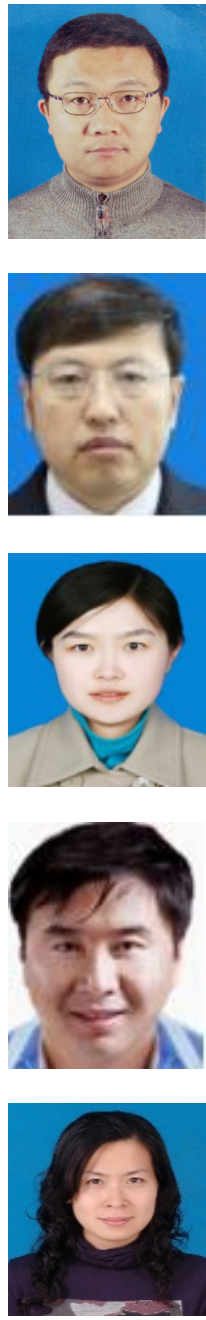

Shuxin Chen current Ph.D. student of fiber engineering in Key Lab of In-fiber Integrated Optics, Ministry Education of China, Harbin Engineering University. Now she works at School of Mechanical Science and Engineering, Qiqihar University. Her current research interests include control algorithm, calculate simulation and fault diagnosis. She is

responsible for the data statistics and analysis.

Yan Liu current Ph.D. student of Chemical Process Machinery in the School of Mechanical Science and Engineering, Northeast Petroleum University. Now he works at Mechanical and Electrical Engineering Institute, Qiqihar University. His current research interests include control, dynamics and fault diagnosis. He is responsible for paper writing and algorithm programming

Jindong Wang received Ph.D. degree in School of Mechanical Science and Engineering, Dalian University of Technology, Dalian, China, in 2000. Now he works at School of Mechanical Science and Engineering, Northeast Petroleum University. His current research interests include control, dynamics and fault diagnosis. He is responsible for presenting the overall framework.

Ying Li current Ph.D. student of Chemical Process Machinery in the School of Mechanical Science and Engineering, Northeast Petroleum University. His current research interests include control, dynamics and fault diagnosis. She is responsible for the thesis pattern recognition algorithm.

Haiyang Zhao received Ph.D. degree in School of Astronautics, Harbin Institute of Technology, Harbin, China, in 2014. Now he works at Northeast Petroleum University. His current research interests include mechanical dynamics and fault diagnosis. He is responsible for experimental vibration test. 